\title{
Creation on the basis of the first theory of strength model equal stressed cylinder exposed to power and temperature loads
}

\author{
Vladimir Andreev ${ }^{1, *}$, and Andrey Leontyev ${ }^{1}$ \\ ${ }^{1}$ National Research Moscow State University of Civil Engineering, 26 Yaroslavskoye Shosse, Moscow, 129337, Russia
}

\begin{abstract}
The paper discusses the axially symmetric plane problem theory of elasticity for inhomogeneous thick-walled cylinder under the action of external pressure and stationary temperature field. The essence of the inverse problem is to determine such dependence of the modulus of elasticity on the radius at which the stress state of the cylinder will be given. It should be noted that an equal stressed cylinder does not coincide with an equal strength cylinder [1] since when the modulus of elasticity of a material changes by modifying its composition, as a rule, its strength properties also change.
\end{abstract}

\section{Introduction}

We assume that in the cylinder is carried out flat strain state. Consider the axisymmetric problem for a radially inhomogeneous cylinder under the action power and temperature loads.

Temperature field caused by the heating of the inner surface of the shell and is stationary. Power load is an external uniform pressure, which creates a prestressed state of the shell.

\section{The resolving equation}

In [2], a resolving equation is given for the stress $\sigma_{r}$ for an axisymmetric problem (plane deformed state) for a radially inhomogeneous body:

$$
\sigma_{r}^{\prime \prime}+\varphi(r) \sigma_{r}^{\prime}+\psi(r)=f(r),
$$

where for the case $v=$ const

$$
\begin{gathered}
\varphi(r)=\frac{3}{r}-\frac{E^{\prime}}{E} ; \psi(r)=-\frac{1-2 v}{r(1-v)} \cdot \frac{E^{\prime}}{E} ; \\
f(r)=-\frac{E(1+v) \varepsilon_{f}^{\prime}}{r\left(1-v^{2}\right)}
\end{gathered}
$$

Here, $E=E(r)$ and $\varepsilon_{f}$ - forced (in this case temperature) strain, which is calculated by the formula:

$$
\varepsilon_{f}=\alpha_{T} T(r),
$$

where $\alpha_{T}=$ const - coefficient of linear temperature expansion.

Substituting (2) and (3) in (1) we obtain the equation:

$$
\sigma_{r}^{\prime \prime}+\left(\frac{3}{r}-\frac{E^{\prime}}{E}\right) \sigma_{r}^{\prime}-\frac{1-2 v}{r(1-v)} \cdot \frac{E^{\prime}}{E} \sigma_{r}=-\frac{E(1+v) \alpha_{T} T^{\prime}}{r\left(1-v^{2}\right)} .
$$

The stationary temperature field in the cylinder, on the inner boundary of which the temperature $T_{0}$ is maintained and on the outer surface $T=0$, is described by the formula:

$$
T(r)=T_{0} \frac{\ln (b / r)}{\ln (b / a)} .
$$

Here $a$ and $b$ - respectively inner and outer radii of the cylinder, $T_{0}=100^{\circ} \mathrm{C}$.

To determine the constants of integration of equation (4) using the boundary conditions

$$
r=a, \quad \sigma_{r}=0 ; \quad r=b, \quad \sigma_{r}=-p_{b}=50 \mathrm{MPa} .
$$

\section{Direct problem for a homogeneous cylinder}

For a homogeneous material, equation (4) is transformed to the form

$$
\sigma_{r}^{\prime \prime}+\frac{3}{r} \sigma_{r}^{\prime}=-\frac{E(1+v) \alpha_{T} T^{\prime}}{r\left(1-v^{2}\right)} .
$$

The solution of equation (7) taking into account (5) and (6) has the form:

$$
\sigma_{r}=\frac{199,95}{2 r^{2}}-99,975+36,05 \ln (r / a) .
$$

From the equation of equilibrium

\footnotetext{
* Corresponding author: asv@mgsu.ru
} 


$$
\frac{d \sigma_{r}}{d r}+\frac{\sigma_{r}+\sigma_{\theta}}{r}=0
$$

we can determine the stresses $\sigma_{\theta}$ :

$$
\sigma_{\theta}=r \sigma_{r}^{\prime}+\sigma_{r}
$$

From the third Duhamel-Neumann relation, setting $\varepsilon_{z}=0$, we can obtain an expression for the stresses $\sigma_{z}$ :

$$
\sigma_{z}=v\left(\sigma_{r}+\sigma_{\theta}\right)-\alpha_{T} E T
$$

Fig. 1 shows the calculation results obtained with the following initial data: $v=0.2 ; a=1 \mathrm{~m} ; b=2 \mathrm{~m}$; $E_{0}=2 \cdot 10^{4} \mathrm{MPa} ; \alpha_{T}=1 \cdot 10^{-5} 1 /{ }^{\circ} \mathrm{C}$.

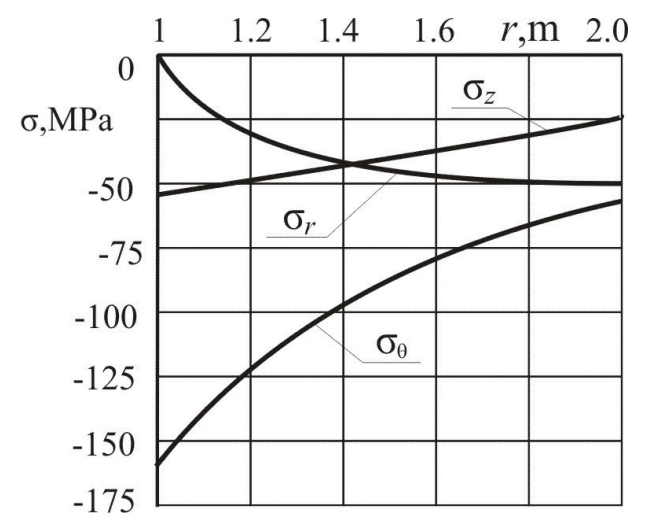

Fig. 1. Stresses in homogeneous cylinder

We note that the solution of the problem was obtained under the assumption that the cylinder is sufficiently long and a flat deformed state is realized in it.

However, in practice, the structures have a finite length and, in the presence of free ends, the stresses $\sigma_{z}$ at the ends will be zero.

As shown in [3], from the condition $\int_{F} \sigma_{z} d A=0$ one can calculate the average stress $\sigma_{z}^{*}=-\int_{F} \sigma_{z} d A / A$, which should be added to the calculated stresses. Then in the middle part of the cylinder there will be a stress state corresponding to the finite cylinder.

\section{Equal stressed cylinder. Inverse problem}

The idea of the method of creating a model of equalstress structures based on the results of numerous calculations inhomogeneous bodies in which it is shown that if in a certain area of the body the modulus of elasticity is less than in a homogeneous material, the stresses in this area are also reduced, and vice versa. Below is the solution of the inverse problem in which wanted the function $E(r)$ decreasing near the inner surface of the cylinder which reduces the stresses in this area. In this case, based on the theory of strength of maximum normal stresses, a stress state is determined that satisfies the condition $\sigma_{\max }=\sigma_{\theta}=$ const, which corresponds to the model of an equally stressed cylinder $[4,5]$. Note that since in this problem all the stresses are negative, then the maximum is considered the largest in absolute value of stresses.

Substituting $\quad \sigma_{\theta}=\sigma_{0}=$ const in equilibrium equation (8) we find

$$
\sigma_{r}^{\prime}=\frac{\sigma_{0}-\sigma_{r}}{r}
$$

The solution of this equation is

$$
\sigma_{r}=\frac{K}{r}+\sigma_{0}
$$

From the boundary conditions (6) one can determine the constants $K$ and $\sigma_{0}: K=100 \mathrm{MPa} \cdot \mathrm{m}, \quad \sigma_{0}=\sigma_{\theta}=-$ $100 \mathrm{MPa}$.

Substituting function (13) in resolving equation (4) after some transformation we find first-order differential equation for determining the function $E(r)$ :

$$
E^{\prime}-\frac{K}{r\left[A(1-k)-k r \sigma_{0}\right]} E-\frac{B}{A(1-k)-\mathrm{kr \sigma}_{0}} E^{2}=0
$$

where

$$
B(r)=-\frac{\alpha_{T} T^{\prime} \cdot r}{1-v}, \quad k=\frac{1-2 v}{1-v} .
$$

Equation (14) is the Bernoulli equation [4], solving it we obtain the desired dependence $E(r)$ :

$$
E=\frac{1}{\frac{B}{a^{m}} \frac{(a r+b)^{n}}{r^{n}} G+\frac{(a r+b)^{n}}{r^{n}} D} .
$$

Here

$$
\begin{aligned}
& G(r)=\ln (a r+b)+\frac{n b}{a r+b}-\frac{n b^{2}}{2(a r+b)^{2}}+\frac{n \mathrm{~b}^{3}}{3(\mathrm{ar}+\mathrm{b})^{3}} \\
& -\frac{b^{n}}{n(a r+b)^{n}} ; a=-k \sigma_{0}, \quad m=n+1, \quad b=K(1-k) .
\end{aligned}
$$

The "reduction of the elastic modulus near the inner contour" mentioned above should be understood not with respect to a homogeneous material, but with respect to the value of $E$ near the outer contour of the cylinder. Therefore, the constant $D$ can be determined from two different boundary conditions for $E$ :

$$
\begin{aligned}
& 1-r=a, E=E_{0}=20000 \mathrm{MПа} \\
& 2-r=b, E=E_{0}=20000 \mathrm{MПа.}
\end{aligned}
$$

In Fig.2. the graphs of the dependence $E(r)$ corresponding to the two indicated variants are presented. 


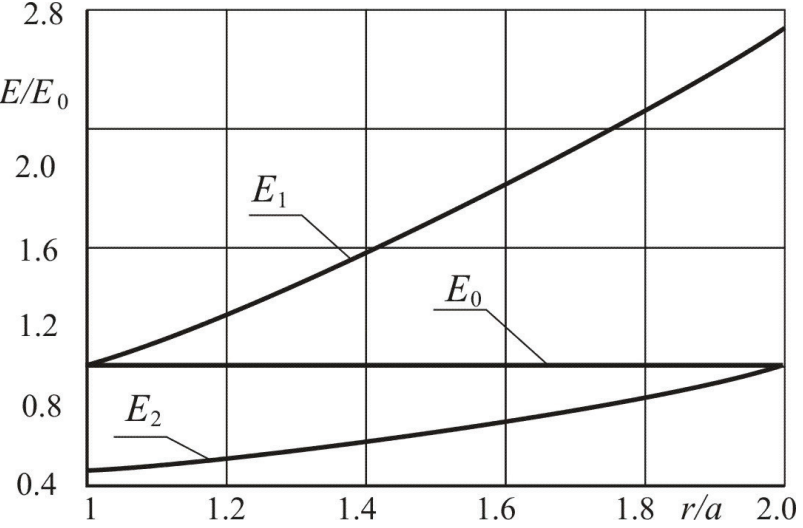

Fig. 2. Dependencies of the elastic modulus in the inhomogeneous $\left(E_{1}, E_{2}\right)$ and homogeneous $\left(E_{0}\right)$ cylinders

To verify the obtained results there was obtained the solution of the direct problem, by direct substitution of the dependence (14) into (4). Fig. 3 shows the stresses diagrams $\sigma_{r}$ and $\sigma_{\theta}$. As was to be expected, the results of stress calculations were the same for both variants. It should be noted that in an equal stressed inhomogeneous cylinder, the stress $\sigma_{\theta \max }^{\text {inhom }}=-100 \mathrm{MPa}$, while in a homogeneous cylinder $\sigma_{\theta \max }^{\text {hom }}=-160 \mathrm{MPa}$. Thus, by introducing a coefficient $\beta=\sigma_{\theta \max }^{\text {hom }} / \sigma_{\theta \max }^{\text {inhom }}$, it is possible to determine how many times the greatest stresses in a homogeneous cylinder are greater than in a similar inhomogeneous one. In this case this coefficient is equal to $\beta=1.6$.

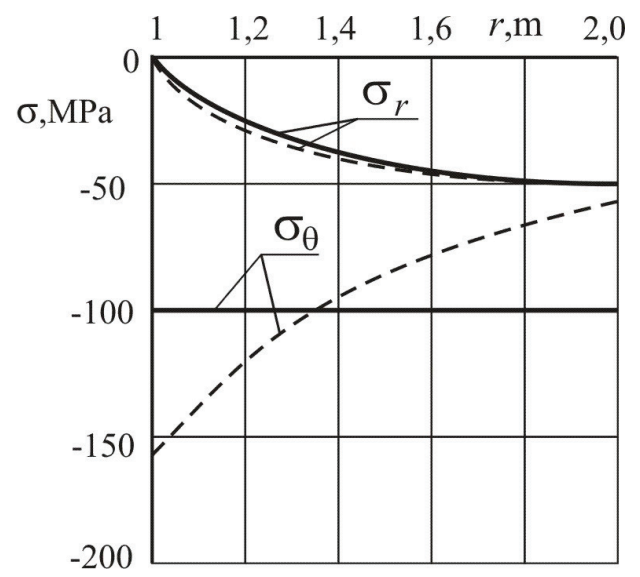

Fig. 3. The stresses in the inhomogeneous ( - ) and homogeneous (- - -) cylinders

Especially it is necessary to stop on stresses $\sigma_{z}$, the diagrams of which are shown in Fig. 4. At first sight, it is surprising difference in stresses $\sigma_{z}$ for two dependencies of $E(r)$ shown in Fig. 2 since the stresses

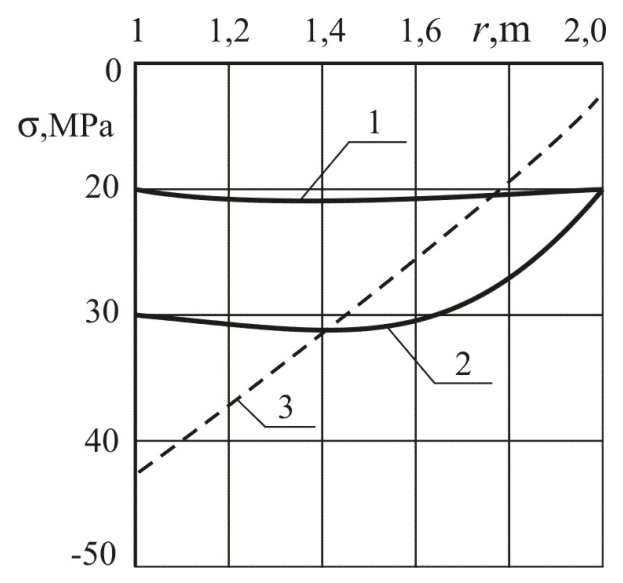

Fig. 4. Stresses $\sigma_{z}$ in a thick-walled cylinder:

1, 2-inhomogeneous cylinder;

3 - homogeneous cylinder

$\sigma_{r}$ and $\sigma_{\theta}$ for these two variants of calculation practically coincides. This fact is explained as follows. Stresses $\sigma_{z}$ for a flat deformed state are calculated from the third Duhamel-Neumann relation (11) which contains the "temperature" term, depending on $E$. If we consider the stresses at the edges of the segment $[a, b]$, then in this example $T(a)=100^{\circ} \mathrm{C}$, and $T(b)=0$. Therefore, at the point $r=b$ due to the absence of a "temperature" term, the stresses coincide, and at the point $r=a$ differ by an amount

$$
\Delta \sigma_{z}=\alpha_{T}\left[E_{1}(a)-E_{2}(a)\right] \cdot T(a) .
$$

Taking into account that $E_{1}(a)=E_{0}=2 \cdot 10^{4} \mathrm{MPa}$, and $E_{2}(a)=0,45 \cdot E_{0}=0,9 \cdot 10^{4} \mathrm{MPa}$, we obtain $\Delta \sigma_{\mathrm{z}}(\mathrm{a})$ $=10 \mathrm{MPa}$, which coincides with the result shown in Fig. 4.

\section{Conclusions}

Models equal stressed cylinders on the basis of other theories strength (maximum shear stress, energy and others) were published in [5-7].

In conclusion, we note that an equal stressed cylinder does not coincide with an equally strength cylinder [3], since the modulus of elasticity of a material changes by modifying its composition, as a rule, its strength properties also change.

There are some materials in which, by modifying the composition, it can be achieved that the modulus of elasticity will vary substantially, and the strength is negligible. Such a material is polymer concrete [8] for which the corresponding mechanical characteristics are given in Table 1. 
Table 1. The physical and mechanical properties of polymer concrete filled with silica flour.

\begin{tabular}{ccc}
\hline $\begin{array}{c}\text { The degree of } \\
\text { filling silica } \\
\text { flour }\end{array}$ & $\begin{array}{c}\text { The modulus of } \\
\text { elasticity } E_{b}, \\
10^{-4} \mathrm{MPa}\end{array}$ & $\begin{array}{c}\text { The ultimate } \\
\text { compressive } \\
\text { strength, MPa }\end{array}$ \\
\hline- & 3.10 & 142 \\
50 & 4.50 & 146 \\
100 & 7.10 & 160 \\
200 & 10.5 & 148 \\
300 & 13.7 & 132 \\
400 & 16.7 & 115 \\
\hline
\end{tabular}

Construction of models of equally strength concrete and reinforced concrete thick-walled cylindrical and spherical shells under the influence of only power loads is devoted to the thesis [9].

The author and his co-workers made attempts to create a thick-walled cylinder with a continuous inhomogeneity of deformation characteristics based on a polymer filled with quartz flour of different factions [10]. During curing, the mold filled with the composite rotated and the filler particles moved towards the outer wall of the mold. Unfortunately, the result was a noncontinuously inhomogeneous cylinder, and a two-layer cylinder whose outer layer was stiffer than the inner layer.

In the above [9], is also considered a model of cylindrical shells, consisting of several layers of different hardness. It is shown that in such models, even with a small number of layers, the efficiency coefficient can reach 1.67 - 1.87 (for a three-layer - five-layer cylinder).

This work was financially supported by the Ministry of Russian Education (state task \#7.1524.2017/PCh)

\section{References}

1. S.P. Timoshenko, J. Goodier, Theory of Elasticity (Moscow, Nauka, 1979)

2. V.I. Andreev, Mechanics of inhomogeneous bodies (Moscow, Publishing House of the ASV, 2002)

3. V.I. Andreev, I.A. Potekhin, Equal stressed and equal strength structures. Modeling and creation, Building materials, equipment, technologies of the XXI century 6 (2009)

4. E. Kamke, Handbook of ordinary differential equations (Moscow, Nauka, 1976)

5. V.I. Andreev, I.A. Potekhin, Construction of a model of an equally stressed cylinder on the basis of the second and fourth strength theory, Proceedings of the 16th Russian-Polish-Slovak seminar "Theoretical Foundations of Construction", Zilina, Slovakia (2007)

6. V.I. Andreev, I.A. Potekhin, Iterative method for constructing a model of an even-strength cylinder, Building Mechanics of Engineering Constructions and Structures, 1 (2008)
7. V.I. Andreev, A.S. Chepurnenko, B.M. Yazyev, Model of Equal-stressed Cylinder based on the Mohr Failure Criterion, Advanced Materials Research. 887-888 (2014)

8. V.V. Paturoev, Polymer concrete (Moscow, Stroyizdat, 1987)

9. V.I. Andreev, I.A. Potekhin, Optimization of the strength of thick-walled shells (Moscow, MGSU, 2011)

10. B.M. Yazyev, V.I. Andreev, R.A. Turusov, Some problems and methods of mechanics of macroinhomogeneous viscoelastic media (Rostov-on-Don, 2009) 